\title{
Design universel : un nouveau paradigme pour l'accessibilité ?
}

Universal design: A new paradigm for accessibility?

Disponible en ligne sur

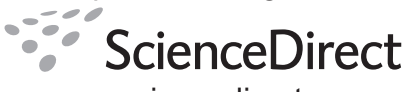

www.sciencedirect.com

\author{
J.-P. Devailly \\ Unité de médecine physique et de réadaptation, CHU d'Avicenne, 125, rue de Stalingrad, \\ $\checkmark 93009$ Bobigny cedex, France
}

es 22 et 23 janvier 2010 se sont tenues à Grenoble les $2^{\mathrm{e}}$ Journées européennes de l'accessibilité. Organisées par l'association " Accès universel ", créée en 2006, elles ont réuni des personnes handicapées, des représentants d'associations, des professionnels impliqués dans tous les aspects de l'accessibilité et dans tous les domaines de la vie en société. Très denses, ces journées ont permis de faire le point sur des avancées majeures dans le domaine de l'architecture, de l'urbanisme, de l'aide à l'emploi, des transports, des voyages, de la communication, des soins et de la culture. L'expérience des nombreux pays européens mais aussi situés hors de l'Europe a été évoquée.

Un nouveau paradigme semble émerger, celui de la conception universelle. La définition figure dans la convention relative aux droits des personnes handicapées adoptée le 13 décembre 2006 par l'Organisation des Nations Unies (OMS) : "On entend par 'conception universelle' la conception de produits, d'équipements, de programmes et de services qui puissent être utilisés par tous, dans toute la mesure possible, sans nécessiter ni adaptation, ni conception spéciale. La " conception universelle " n'exclut pas les appareils et accessoires fonctionnels pour des catégories particulières de personnes handicapées là où ils sont nécessaires " [1]. Le principe est simple : ce qui est indispensable à quelques personnes handicapées est nécessaire pour beaucoup plus et confortable pour tous. L'accessibilité donne une " haute qualité d'usage " pour tous au domaine qui est alors rendu accessible. Chacun le comprendra aisément en pensant au métro, avec l'exemple du métro japonais, adapté pour les déficiences physiques, cognitives, pour les personnes nonvoyantes et malentendantes avec ses dalles en braille, ses icônes intuitives, ses systèmes d'orientation pour sourds. Une idée forte est aussi que le développement des systèmes

e-mail : jpdevailly@gmail.com. d'accès universel réduit tellement les coûts de production par leur utilisation massive qu'il n'y a pas en cela contradiction entre coûts et gain de qualité de vie. On peut citer ici les exemples des télécommandes, des systèmes de dictée vocale, de la ville rendue accessible pour les voyageurs lourdement chargés de valises à roulettes, les mères avec landaus, les blessés portant temporairement des plâtres et marchant avec des cannes, les gens poussant leur vélo ou leur scooter dans les escaliers des gares, enfin celui de l'ouverture massive de transports et de loisirs rendus plus accessibles à l'immense marché des personnes âgées.

Le design universel est réussi quand il est invisible, il rend aussi le handicap moins visible en supprimant les barrières environnementales qui en sont la cause. Toutefois, comme toute révolution culturelle annoncée, ce concept peut aussi faire craindre quelques limites. L'accessibilité universelle estelle vraiment applicable à tous les domaines du handicap ? II suffit de penser aux difficultés croissantes pour les personnes ayant des troubles cognitifs à s'orienter et se faire accepter dans une cité toujours plus complexe. L'invisibilité souhaitée du handicap, qui va jusqu'à vouloir exclure tous les mots de l'exclusion, même ceux d'intégration et d' " inclusion ", estelle souhaitée par les premiers intéressés ? "Mal nommer les choses, c'est ajouter au malheur du monde ", disait Camus. Plusieurs intervenants ont rappelé la nécessité " d'appeler un chat un chat " pour parvenir à trouver des méthodes et des objectifs partagés. La " maîtrise d'usage " tant recherchée suppose une bonne identification de l'usager qui sera partie prenante d'un projet. La conception universelle, voire l'« usage universel " sont des concepts féconds dans la perspective du développement humain et durable. Cependant, l'obstacle à supprimer, qui constitue le fondement de cette vision de la lutte contre le handicap en en faisant un état que l'on pourrait faire disparaître par la seule action sur les systèmes politiques, ne doit pas faire oublier l'ensemble des dimensions biopsy- 
chosociales d'un "processus de production du handicap " contre lesquelles la prévention doit s'acharner. Ici, le lien doit se retisser entre système de soins et monde du handicap dans le continuum proposé par l'OMS : prévention, soins curatifs, réadaptation et accompagnement [2].

L'oubli de ce processus et de sa prévention à chaque étape d'une chaîne complexe de cascades lésionnelles, fonctionnelles et situationnelles risquerait d'avoir de très lourdes conséquences dans le contexte de la " nouvelle gestion publique ". Le besoin qui n'est pas identifié n'aura pas d'indicateur, sans indicateur il ne sera pas financé et des défaillances majeures risquent d'en résulter. Car dans le domaine de la santé, les nouveaux modèles de régulation supposent non pas l'accès à tout pour tous mais la détermination du juste soin au juste coût. Le poids des systèmes sur les rationalités limitées des acteurs pose ici les limites d'actions qui seraient surtout centrées sur la formation, la sensibilisation, la pédagogie, voire la contrainte sans s'interroger simultanément sur l'impact des modèles de gestion sur l'autonomie réelle des professionnels et des usagers [3].

Le concept de "pouvoir médical " concentre les représentations négatives des limites de la médecine, de ses modèles mentaux et de son manque trop fréquent d'humilité et d'écoute. Il focalise des expériences épouvantables d'usagers handicapés et de leurs proches avec le système de soins et l'on pense aux témoignages poignants et acerbes de parents d'autistes ou de schizophrènes à l'égard de la psychanalyse. Hors cet exemple qui touche la psychiatrie, mais qu'on pourrait décliner partout ailleurs, tous les domaines de la médecine doivent être à l'écoute de ce savoir des usagers. Mais la critique d'un modèle biomédical jugé trop envahissant s'est radicalisée dans la confusion entre médecine sociale et la médicalisation constitutive du biopouvoir tel que le décrivait Michel Foucault [4].

Ainsi a émergé une anti-médecine militante, alimentée à des sources aussi diverses que la biopolitique de Foucault, la Némésis médicale d'Illitch et l'approche sociologique d'une profession aux prétentions jugées illégitimes jusqu'à ce que le revirement d'Eliot Freidson' le conduise à prôner l'autorégulation professionnelle comme " troisième logique " indispensable pour équilibrer dans le monde de la santé les régulations marchandes et le managérialisme bureaucratique [5]. Mais cette spirale de la défiance a laissé progressivement émerger l'idée qu'il fallait démédicaliser le social à outrance au risque conjoint de l'oubli de la prévention (aucune occurrence dans le rapport de l'ONU) et de l'aggravation paradoxale de l'inaccessibilité aux soins. Elle a engendré, pour les populations les plus fragiles, des dégâts humains, sanitaires et économiques considérables liés à la fragmentation grandissante des continuums de soins entre secteurs sanitaire et social, compliquée

\footnotetext{
${ }^{1}$ Freidson présente trois logiques de régulation des activités professionnelles : le contrôle par le marché, le contrôle bureaucratique et le contrôle professionnel dénommé "professionnalisme".
}

de l'empilement en mille-feuille de dispositifs successifs de guichets de niveaux stratégiques et de financements, malgré les incantations officielles.

Le dernier échec patent de cette production administrative de l'inaccessibilité par la défiance est celui de l'illusion d'un réseau centralisé autour du guichet unique des Maisons départementales des personnes handicapées, par lequel certaines associations d'usagers souhaitaient échapper à l'emprise sanitaire en s'associant aux managers dont la main devient cependant de plus en plus visible [6]. On a ainsi éparpillé dans un labyrinthe qu'aucun fil d'Ariane ne vient adoucir les compétences qu'il fallait assembler, disloqué les réseaux informels et les savoir-faire de nombreux collectifs professionnels, laissant les personnes les moins habiles toujours plus démunies face à une injonction d'autonomie qui, dans ce contexte, ne pouvait conduire qu'à majorer le handicap [7].

Prenons garde à ce que la conception universelle ne conduise pas à l'oubli de ce qui doit être spécifiquement organisé pour la prévention et la réduction du handicap. Elle deviendrait alors l'arbre qui cache la forêt d'une inaccessibilité croissante aux soins, masquant l'impérieuse nécessité de mieux intégrer les parcours de soins et d'accompagnement entre soins et social. La fragmentation gestionnaire et la myopie de ses indicateurs risque fort, malgré les effets encore incertains des agences régionales de santé, de désocialiser encore davantage le sanitaire en même temps qu'elle démédicalise le social. N'oublions surtout pas que la médecine a toujours été aussi un contre-pouvoir indispensable dans la cité, qui doit déployer une inlassable rhétorique contre les sophistes et les charlatans, portée par la certitude que les médecins ont de devoir être, quand il le faut, les avocats de la santé de leurs patients.

Il reste à fonder en raison les bénéfices apportés par l'accessibilité et la lutte contre le handicap pour mieux lancer des défis aux décideurs publics et privés. Ce qui émerge avec force de ces journées de l'accessibilité, c'est que le devoir d'optimisme et l'espoir doivent prévaloir. Le monde des soins et de l'action économique et sociale en général doit viser une stratégie intégrée. Les différentes parties prenantes (usagers, professionnels, élus, gestionnaires et monde marchand) se proposent pour challenger les décideurs, de co-construire un cadre de référence partagé pour un objectif commun : réduire le handicap quelle qu'en soit l'origine et à n'importe quel âge.

\section{Conflit d'intérêt}

Aucun.

\section{Références}

[1] Convention relative aux droits des personnes handicapées. Organisation des Nations Unies 2006 [cité 2010 Feb 25]. Dis- 
ponible sur : URL : http://www.un.org/disabilities/documents/ convention/convoptprot-f.pdf.

[2] Incapacités, prévention, traitement et réadaptation compris. Organisation Mondiale de la santé, rapport du secrétariat, 14 avril 2005 [cité 2010 Feb 25]. Disponible sur : URL : http:// www.who.int/gb/ebwha/pdf_files/WHA58/A58_17-fr.pdf.

[3] Dupuis A, Farinas L. Une critique des modes managérialistes dans la gestion des organisations de services humains complexes de santé et de services sociaux. Cahier de recherche du Cergo 2009 Feb [cited 2010 Feb 25]. Disponible sur : URL : http://benhur.teluq.uquebec.ca/SPIP/cergo/IMG/pdf/Cahier_ du_Cergo_2009-02.pdf.

[4] Renault E. Biopolitique, médecine sociale et critique du libéralisme. Multitudes 2008; 4(34):195-205. Disponible sur : URL : http://www.cairn.info/load pdf.php?ID ARTICLE= MULT_034_0195.

[5] Freidson E. Professionalism, the third logic. In: On the practice of knowledge. Chicago: The University of Chicago Press; 2001.

[6] Piovesan D, Robelet $M$, Claveranne JP. La main visible des managers associatifs : les transformations en cours du marché et des organisations dans le secteur du handicap Communication à la 13 journée d'étude du GDR CADRES CENS - CAR CEREO Pays de la Loire - MSH Ange Guépin, 19 octobre 2007; Nantes. Disponible sur : URL : http://gdr-cadres.cnrs.fr/pdf/GDR13-2 \%2oPiovesan.pdf.

[7] Ebersold S. Le champ du handicap, ses enjeux et ses mutations : du désavantage à la participation sociale. Análise Psicológica 2002;20(3):281-90. 